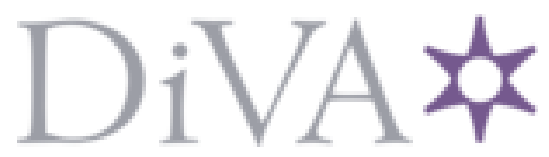

http://www.diva-portal.org

This is the published version of a paper presented at IEEE International Ultrasonics Symposium.

Citation for the original published paper:

Widman, E., Larsson, M., Caidahl, K., Heyde, B., D’hooge, J. (2013)

Speckle Tracking Strain Estimation of a Carotid Artery Plaque Phantom - Validation via

Sonomicrometry.

In: IEEE conference proceedings

N.B. When citing this work, cite the original published paper.

Permanent link to this version:

http://urn.kb.se/resolve?urn=urn:nbn:se:kth:diva-137662 


\title{
Speckle Tracking Strain Estimation of a Carotid Artery Plaque Phantom - Validation via Sonomicrometry
}

\author{
Erik Widman $^{1}$, Kenneth Caidahl ${ }^{2}$, Brecht Heyde $^{3}$, Jan D’hooge ${ }^{3}$, Matilda Larsson ${ }^{1,3}$ \\ ${ }^{1}$ Department of Medical Engineering, School of Technology and Health, KTH Royal Institute of Technology, Stockholm, Sweden \\ ${ }^{2}$ Department of Molecular Medicine and Surgery, Karolinska Institutet, Stockholm, Sweden \\ ${ }^{3}$ Cardiovascular Imaging \& Dynamics, Department of Cardiovascular Sciences, KU Leuven, Leuven, Belgium
}

\begin{abstract}
Current clinical ultrasound-based methods for plaque characterization are limited to visual assessment of plaque echogenicity creating demand for quantitative diagnostic tools. Our objective was to validate radial and longitudinal speckle tracking (ST) strain in phantom plaques via sonomicrometry (sono), and to compare the peak plaque and arterial wall strain. Four carotid artery gel-phantoms with a soft wall inclusion, mimicking a vulnerable plaque, were constructed. The phantoms were connected to a programmable pump simulating a carotid flow. Cineloops were acquired using a GE Vivid E9 where radial and longitudinal strain were calculated using a normalized crosscorrelation ST algorithm. The region of interest was adjusted according to the plaque size. Sonomicrometry was used as a reference measurement. The correlation between estimated mean peak strain and the reference peak strain was $r=0.96(p<0.001)$ radially and $r=0.75(p \leq 0.005)$ longitudinally. The soft plaque exhibited 35.1\% (SD 16.9\%) greater radial $(p<0.001)$ and $88.6 \%$ (SD 72.0\%) greater longitudinal $(p<0.001)$ peak strain than the arterial wall when measured with speckle tracking. It was possible to estimate plaque strain by $\mathrm{ST}$ and to distinguish a soft plaque from the vessel wall via strain measurements.
\end{abstract}

Keywords-Ultrasound; Speckle Tracking; Plaque; Strain; Phantom; Sonomicrometry; Carotid Artery

\section{INTRODUCTION}

Vulnerable plaques have a high probability of rupture causing emboli to break off from the plaque and travel via the circulatory system to vessels in the brain. As the arteries become smaller the emboli can lodge in the vessel wall restricting the blood flow to parts of the brain yielding a temporary transient ischemic attack or permanent thrombotic stroke [1]. Plaque characterization in the carotid artery is therefore critical in risk assessment for cerebral ischemic events. In clinical practice, ultrasound-based methods for plaque characterization are limited to visual assessment and evaluation of plaque morphological and echogenic properties [2]. Treatment options, such as an endarterectomy or angioplasty, are often based on plaque morphology, hypoechoic area, or echo reflection in the plaque [3, 4]. Quantitative tools are needed to better assess the likelihood of plaque rupture to determine if invasive treatment options are required.

Identification and characterization of vulnerable plaques via intravascular ultrasound have been previously attempted $[5,6]$ as well as characterization by radial strain and attenuation coefficient [7]. However, extensive validation via reference methods are still missing. Recently, our group published a speckle tracking algorithm successfully estimating strain in the wall of a carotid artery phantom [8]. The aim of this study was to use the same speckle tracking algorithm to validate radial and longitudinal strain in plaques via sonomicrometry, and compare the measured plaque and arterial wall strain.

\section{METHODS}

\section{A. Phantom Construction}

Four in-vitro carotid artery phantoms with a plaque mimicking inclusion were constructed from a mixture in mass percent of $87 \%$ deionized water, $10 \%$ polyvinyl alcohol (PVA) with a molecular weight of $56.140 \mathrm{~g} / \mathrm{mol}$ (SigmaAldrich, St. Louis, MO) and 3\% graphite powder with particle size < $50 \mu \mathrm{m}$ (Merck KGaA, Darmstadt, Germany). The solution was heated and stirred until the mixture thickened and was fully dissolved. It was subsequently poured in the vessel phantom mold, which consisted of a hollow acrylic block (cylindrical shape with a $12 \mathrm{~mm}$ diameter, $100 \mathrm{~mm}$ length, and molded fixing collars) and a bronze rod with a $6 \mathrm{~mm}$ diameter placed in the center of the hole. The metallic rod had a $50 \mathrm{~mm}$ long and $1.5 \mathrm{~mm}$ thick extension attached to create a cavity in the phantom wall (Fig. 1a).

Once the solution was poured in the mold, it stayed confined between the acrylic block and the rod, resulting in a tube-shaped phantom. The phantom was frozen for 12 hours at approximately $-23{ }^{\circ} \mathrm{C}$ and then thawed for 12 hours at room temperature $\left(\approx 20{ }^{\circ} \mathrm{C}\right)$. This freeze-thaw cycle was repeated twice for three phantoms and three times for one of the phantoms. Subsequently, the wall cavity was filled with a PVA-graphite solution, prepared the same way as previously described, and the entire phantom was exposed to one extra freeze-thaw cycle, resulting in three and four freeze-thaw cycles for the vessel wall and one for the cavity mimicking the plaque. Since PVA stiffens with increasing freeze-thaw cycles, the phantom wall became stiffer than the inclusion, mimicking the vessel wall and the soft plaque, respectively. A schematic representation and photo of the phantom are shown in Fig. 1. Consequently, as the plaque cavity was filled with PVA the thin vessel wall $(0.75 \mathrm{~mm})$ extruded into the lumen creating a realistic partial lumen occlusion shown in Fig. 2a. 


\section{B. Experimental Setup}

The PVA-phantoms where attached to a polyvinyl chloride (PVC) box $(100 \mathrm{~mm} \times 85 \mathrm{~mm} \times 300 \mathrm{~mm})$ by placing two plastic discs around the fixing collars of the phantoms and tightening them to the fixture with screws. A mixture of $3 \%$ agar (Merck KGaA, Darmstadt, Germany), 4\% graphite power, and $93 \%$ deionized water by mass was heated while continuously stirred to $73{ }^{\circ} \mathrm{C}$, then cooled to $40{ }^{\circ} \mathrm{C}$, and subsequently poured into the PVC-box until the PVAphantom was covered with approximately $1 \mathrm{~cm}$ of agarmixture.

The phantoms were connected to a programmable pump (CompuFlow 1000MR; Shelley Medical Imaging Technologies, Ontario, Canada) by attaching hoses with a $6 \mathrm{~mm}$ inner diameter to plastic disks that attached the phantom to the PVC-box by compression. The pump was programmed to simulate a carotid flow profile at a rate of 60 cycles/min. A solution of $40 \%$ glycerin (Merck KGaA, Darmstadt, Germany) and $60 \%$ deionized water mixture was pumped through the phantom at peak flow rates 10, 20, 30, and $35 \mathrm{~mL} / \mathrm{s}$. The pump was purged before the experiments to remove air bubbles from the blood-mimicking fluid.

\section{Data Aquisition}

Sonomicrometry data and ultrasound cineloops of the plaque, as well as the vessel wall, were collected at every flow rate for each phantom. Sonomicrometry data were collected for $30 \mathrm{~s}$ as a reference strain using a digital Sonometrics (Ontario, Canada) acquisition system sampling at $200 \mathrm{~Hz}$. Sonomicrometry crystals $1 \mathrm{~mm}$ in diameter were super-glued (Loctite, Düsseldorf, Germany) to the plaque lumen and posterior phantom wall (Fig. 1a). Ultrasound image acquisition was turned off during sonomicrometry data collection to avoid sound interference.

Ultrasound long-axis images were collected on a GE Vivid E9 ultrasound machine (Horten, Norway) with a fixed 9LD linear array transducer. Long-axis cineloops were recorded throughout 3 cycles for each flow rate at a frame rate of 42 $\mathrm{Hz}$, center frequency $10 \mathrm{MHz}$, and a $3.5 \mathrm{~cm}$ depth with image compounding turned off. Fig. 2a shows a sample B-mode long-axis picture of the phantom with plaque inclusion embedded in the agar mixture. A schematic of the experimental setup is shown in Fig. 2 b.

\section{Data Analysis}

The collected sonomicrometry and B-mode data were analyzed in Matlab 2011a and synchronized by recording a simulated electrocardiogram (ECG) signal from the CompuFlow pump. An in-house developed speckle tracking algorithm calculated the peak plaque strain which was compared to the strain found from the movement recorded by the sonomicrometry crystals.

\section{1) Speckle Tracking Analysis}

A previously described algorithm [9] based on normalized cross-correlation was used to perform the speckle tracking analysis. Axial (along the ultrasound beam) and lateral (orthogonal to the ultrasound beam) speckle tracking was performed on 3 consecutive pump cycles with a kernel length a)

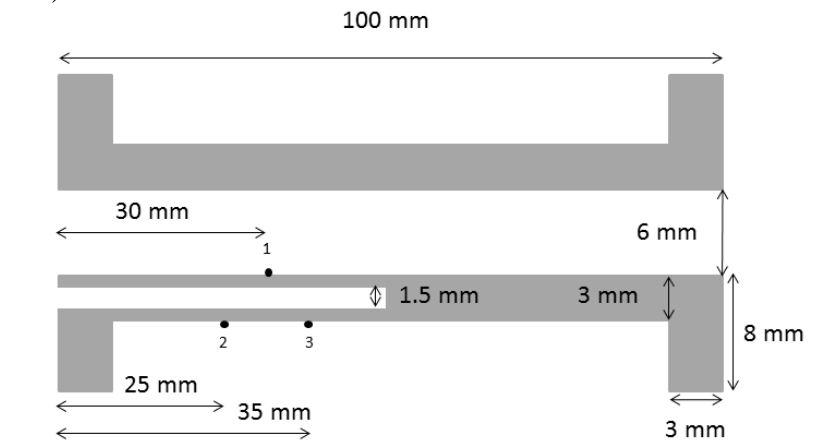

b)

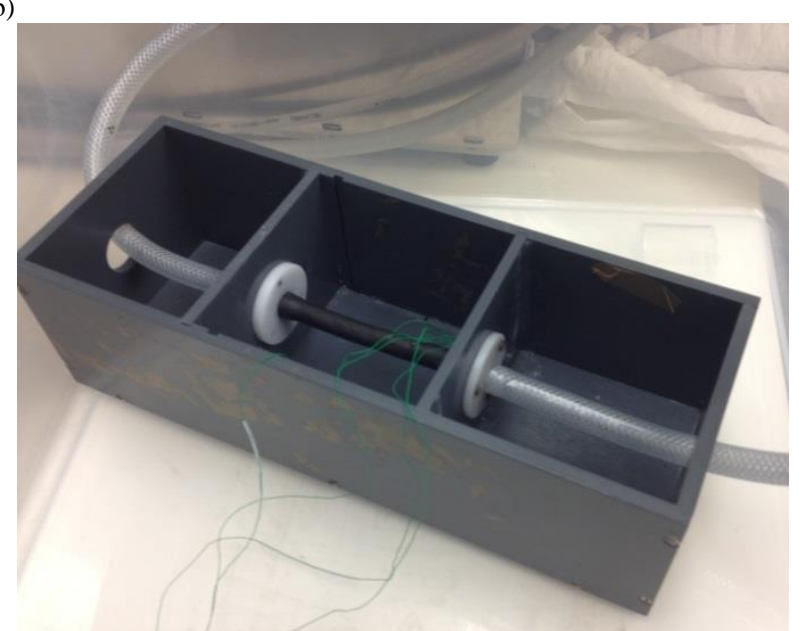

Fig. 1. a) Long-axis cross-sectional schematic illustration of the phantom with dimensions and placement of sonomicrometry crystals (1-3). b) Photo of the vessel phantom attached to the polyvinyl chloride (PVC) box connected to the pump prior to filling the cavity with the agar-mixture. The wires are leads to the sonomicrometry crystals.

and width of 3 and 5 wavelengths $(\lambda)$ respectively. The kernel was moved in a search area which was defined to allow maximum velocities of $2 \mathrm{~cm} / \mathrm{s}$ with a $60 \%$ axial and lateral overlap using spline interpolation to detect sub-sample motion. The speckle tracking strain curves were then obtained by linear regression in a region of interest (ROI) and subsequently low pass filtered with an averaging window 3 samples in length to remove noise.

\section{a) Plaque Strain Estimation}

The ROI size was based on measurements of the plaque size measured in diastole. The ROI was manually selected near the vertical center of the plaque, offset to the right of the sonomicrometry crystal in the lumen to avoid effects of shadowing. The ROI for radial strain was selected to be approximately $50 \%$ of the plaque height and $1.5 \mathrm{~mm}$ in width. The longitudinal ROI width was approximately $50 \%$ of the distance from the crystal to the apex of the plaque (typically $4 \mathrm{~mm}$ width) and $1.5 \mathrm{~mm}$ in height.

\section{b)Vessel Wall Strain Estimation}

Two of the phantoms were used for comparison between vessel wall and plaque strain. The vessel wall ROI was placed on the same posterior side of the phantom as the plaque to achieve similar boundary conditions from the PVC-box and 
a)

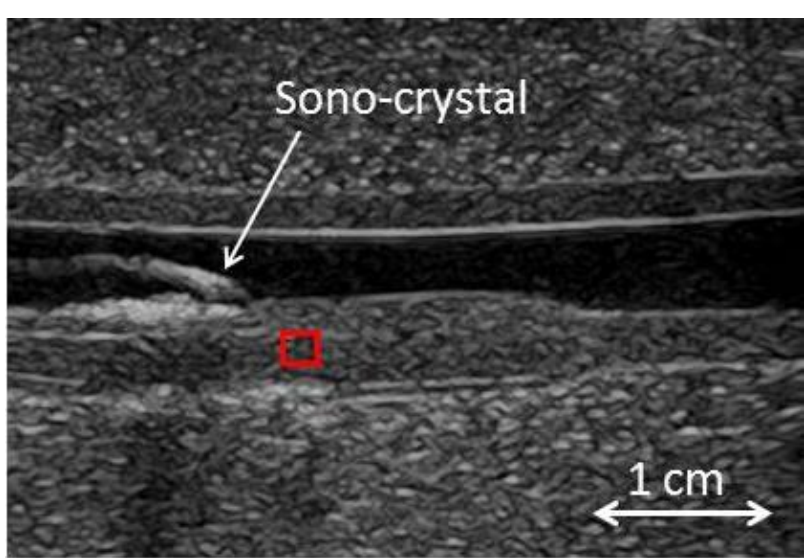

b)

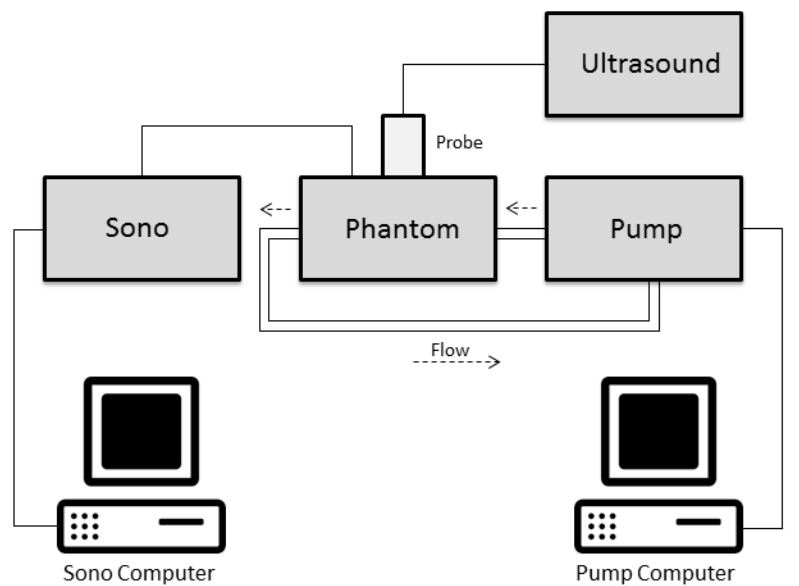

Fig. 2.a) B-mode long-axis image of phantom vessel with soft plaque inclusion to the left posterior wall. The red box indicated the region of interest (ROI) for the speckle tracking radial strain analysis offset to the right of the lumen sono-crystal to avoid the shadowed region. b) Diagram of the experimental setup illustrating connections between the phantom, sonomicrometry system, programmable pump, and ultrasound machine.

agar-mixture. The ROI was manually placed approximately $2 \mathrm{~cm}$ from the plaque in the center of the vessel wall with a $1.5 \times 1.5 \mathrm{~mm}$ radial and $5 \times 1.5 \mathrm{~mm}$ longitudinal ROI size.

\section{2) Sonomicrometry}

Outliers from the inter-crystal displacement curves were removed by thresholding, followed by filtering the resulting signals with a median filter 10 samples in length. Five cycles were averaged to obtain the final reference displacement curves. The radial and longitudinal strain was calculated according to previously described methods [9].

\section{E. Speckle Tracking Sonomicrometry Comparison}

The root mean square error (RMSE) was calculated on the first cycle of B-mode data for each flow condition per phantom as

$$
\operatorname{RMSE}=\sqrt{\sum_{\mathrm{n}=1}^{\mathrm{N}} \frac{(\varepsilon(\mathrm{n})-\hat{\varepsilon}(\mathrm{n}))^{2}}{\mathrm{~N}}}
$$

where $\varepsilon(n)$ is the reference sonomicrometry strain, $\hat{\varepsilon}(n)$ is the estimated speckle tracking strain, and $\mathrm{N}$ is the number frames in one pump cycle. The bias was calculated as the absolute difference between the reference and estimated peak systolic strain $\left(\left|\varepsilon_{\text {peak }}-\widehat{\varepsilon}_{\text {peak }}\right|\right)$ and averaged for all flow conditions and phantoms. Bland-Altman plots for sonomicrometry and speckle tracking peak strain were created and correlation analysis was performed. A paired t-test was performed to compare the plaque and the wall strain.

\section{RESULTS}

Sample speckle tracking and sonomicrometry plaque strain curves for radial and longitudinal strain can be seen in Fig. 3a and Fig. 3c respectively. Fig. 3b (radial) and Fig. 3d (longitudinal) show correlation plots of speckle tracking versus sonomicrometry for radial $(r=0.96, p<0.001)$ and longitudinal $(\mathrm{r}=0.75, \mathrm{p} \leq 0.01)$ peak strain. The radial and longitudinal Bland-Altman plots can be seen in Fig. 4a and Fig. 4b respectively. Table I shows the mean and standard deviation for the bias and RMSE for the radial and longitudinal strain. Longitudinal strain data was discarded for one of the phantoms due to irregular movement caused by fluid in the plaque cavity. The plaque exhibited $35.1 \%$ (SD $16.9 \%$ ) greater radial $(\mathrm{p}<0.001)$ and $88.6 \%$ (SD 72.0\%) greater longitudinal $(\mathrm{p}<0.001)$ peak strain than the arterial wall when measured with speckle tracking.

\section{DISCUSSION}

This validation study indicates the feasibility of assessing radial and longitudinal strain in atherosclerotic plaques. A strong correlation between speckle tracking and reference strain values was found radially (Fig. 3b), while the agreement in the longitudinal direction (Fig. 3d) was not as strong but still good. This is most likely due to the lower resolution in the lateral direction coupled with little longitudinal plaque movement. The radial and longitudinal bias and RMSE (Table I) indicates less absolute error in the longitudinal strain. However, it must be taken into consideration that the radial a)

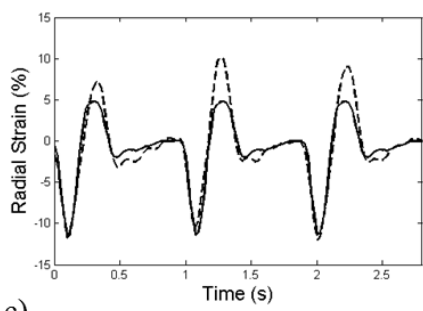

c)

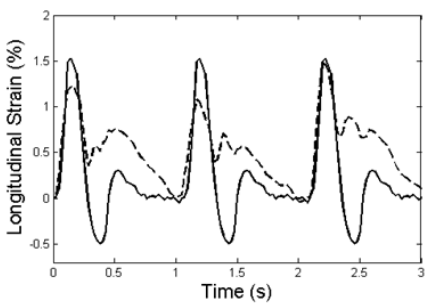

b)

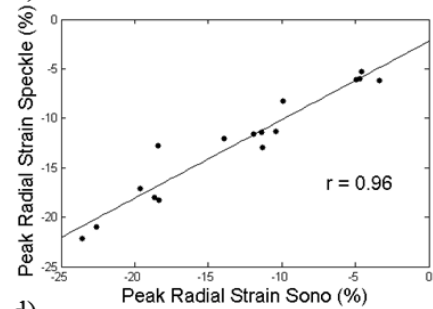

d)

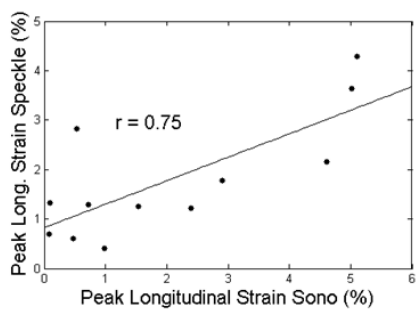

Fig. 3. Sample strain curves for sonomicrometry (solid line) and speckle tracking (dashed line) for radial (a) and longitudinal (c) directions. Correlation plots for peak speckle tracking strain versus peak sonomicrometry strain for radial (b) and longitudinal (d) directions along with correlation values. 
a)

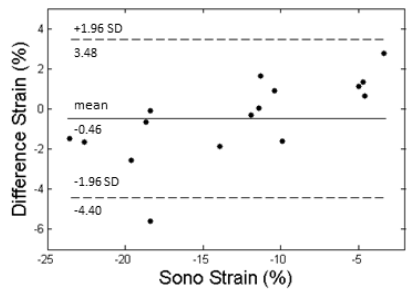

b)

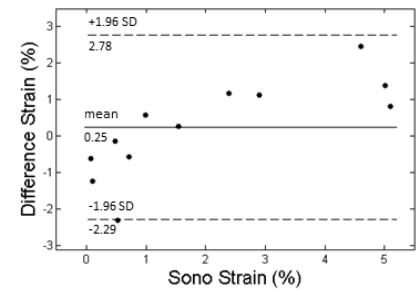

Fig. 4. Radial (a) and longitudinal (b) Bland-Altman plots showing the difference between the sonomicrometry and speckle tracking strain versus the sonomicrometry strain.

TABLE I. STRAIN ESTIMATION RESULTS FROM SPECKLE TRACKING ANALYSIS IN THE PLAQUE. RADIAL AND LONGITUDINAL DIFFERENCE BETWEEN PEAK REFERENCE AND ESTIMATED STRAIN (BIAS) AS WELL AS THE RMSE.

\begin{tabular}{|lcc|}
\hline & $\begin{array}{c}\text { Radial strain } \\
\text { Mean }(\text { SD })\end{array}$ & $\begin{array}{c}\text { Longitudinal strain } \\
\text { Mean }(\text { SD })\end{array}$ \\
\hline Bias $(\%)$ & $2.09( \pm 2.03)$ & $1.61( \pm 1.57)$ \\
RMSE $(\%)$ & $2.93( \pm 2.18)$ & $0.78 \pm 0.49)$ \\
\hline
\end{tabular}

*SD $=$ Standard Deviation, RMSE $=$ Root Mean Square Error

strain is significantly larger in magnitude than the longitudinal strain. The radial Bland-Altman plot (Fig. 4a) shows that the speckle tracking algorithm overestimates low strain values and underestimates high values. The inverse is true for longitudinal strain. Radially, the limits of agreement (LOA) are acceptable in relation to the range whereas the longitudinal LOA are higher as a result of two outliers (Fig. 4b). However, there is still good longitudinal correlation $(r=0.75)$ between sonomicrometry and speckle tracking.

Creating a phantom with a soft plaque inclusion was challenging to successfully manufacture resulting in several design modifications. Eight phantoms were attempted of which only four succeeded. The first two successful phantoms leaked fluid into the plaque cavity when connected to the pump. This resulted in less radial and longitudinal plaque strain combined with longitudinal plaque compression in one of the phantoms. Furthermore, the fluid degraded the image quality of the plaque. The final two phantoms were modified by super-gluing the plaque cavity shut after the final freeze cycle to prevent fluid from the lumen to leak into the cavity. These phantoms were used for the strain comparison between plaque and vessel wall.

A second source of error was the placement of the lumen sonomicrometry crystal on the plaque (Fig. 1a crystal 1). Limited visibility and accessibility made it difficult to place the crystal on the center of the lumen plaque extrusion. Offcenter placement can result in under estimation of radial displacement. The fairness of comparing sonomicrometry and speckle tracking can also be questioned since the crystals and ROI were not placed at the exact same location in the plaque to avoid the shadowed region under the crystals (Fig. 2a).

The phantom plaque radial strain had a significant gradient where the movement during the cardiac cycle of the plaque tissue near the lumen is much greater than the tissue near the phantom edge. To avoid these extremes, the ROI was placed in the center of the plaque combined with limiting the ROI height to $50 \%$ of the vertical plaque size in order to get an average value for the plaque strain. It should also be noted that in this experimental setup a plaque in the common carotid artery was mimicked, but it is more common for plaque buildup to occur in artery bifurcation [10].

Despite these limitations, the results of the two methods are in good agreement and it is possible to distinguish the plaque from the arterial wall. These results are in line with other studies showing it is feasible to measure plaque strain invivo [5]. Furthermore, Schaar [6] found a high sensitivity $(88 \%)$ and specificity (89\%) when characterizing plaque with strain using intravascular ultrasound. Further studies, including an in-vivo study and an in-vitro excised plaque study, are necessary to test the algorithm on a patient population and determine its sensitivity in distinguishing hard and soft plaque.

\section{CONCLUSION}

This study shows the feasibility of radial and longitudinal strain estimation in a carotid artery plaque phantom which has been validated by sonomicrometry. Additionally, the ability to distinguish between a soft plaque and vessel wall in a phantom has been shown.

\section{REFERENCES}

[1] I. P. Reckless and A. M. Buchan, "Stroke: management and prevention," Medicine, vol. 36, pp. 592-600, 11// 2008.

[2] U. Prahl, P. Holdfeldt, G. Bergstrom, B. Fagerberg, J. Hulthe, and T. Gustavsson, "Percentage white: a new feature for ultrasound classification of plaque echogenicity in carotid artery atherosclerosis," in Ultrasound in Medicine and Biology. vol. 36, ed England, 2010, pp. 21826.

[3] A. C. Gray-Weale, J. C. Graham, J. R. Burnett, K. Byrne, and R. J. Lusby, "Carotid artery atheroma: comparison of preoperative B-mode ultrasound appearance with carotid endarterectomy specimen pathology," J Cardiovasc Surg (Torino), vol. 29, pp. 676-81, Nov-Dec 1988.

[4] S. K. Kakkos, M. B. Griffin, A. N. Nicolaides, E. Kyriacou, M. M. Sabetai, T. Tegos, et al., "The size of juxtaluminal hypoechoic area in ultrasound images of asymptomatic carotid plaques predicts the occurrence of stroke," Journal of Vascular Surgery, vol. 57, pp. 609-617, Mar 2013.

[5] C. L. de Korte, M. J. Sierevogel, F. Mastik, C. Strijder, J. A. Schaar, E. Velema, et al., "Identification of atherosclerotic plaque components with intravascular ultrasound elastography in vivo: a Yucatan pig study," Circulation, vol. 105, pp. 1627-30, Apr 92002.

[6] J. A. Schaar, C. L. De Korte, F. Mastik, C. Strijder, G. Pasterkamp, E. Boersma, et al., "Characterizing vulnerable plaque features with intravascular elastography," in Circulation. vol. 108, ed United States, 2003, pp. 2636-41.

[7] H. Shi, T. Varghese, R. J. Dempsey, M. S. Salamat, and J. A. Zagzebski, "Relationship between ultrasonic attenuation, size and axial strain parameters for ex vivo atherosclerotic carotid plaque," in Ultrasound Med Biol. vol. 34, ed England, 2008, pp. 1666-77.

[8] M. Larsson, F. Kremer, P. Claus, T. Kuznetsova, L. A. Brodin, and J. D'hooge, "Ultrasound-Based Radial and Longitudinal Strain Estimation of the Carotid Artery: A Feasibility Study," IEEE Transactions on Ultrasonics Ferroelectrics and Frequency Control, vol. 58, pp. 22442251, Oct 2011

[9] M. Larsson, F. Kremer, B. Heyde, L. A. Brodin, and J. D'hooge, "Ultrasound-based Speckle Tracking for 3D Strain estimation of the Arterial wall - An experimental validation study in a tissue mimicking phantom," 2011 IEEE International Ultrasonics Symposium (Ius), pp. 725-728, 2012.

[10]C. Oviedo, A. Maehara, G. S. Mintz, H. Araki, S. Y. Choi, K. Tsujita, et al., "Intravascular ultrasound classification of plaque distribution in left main coronary artery bifurcations: where is the plaque really located?," in Circ Cardiovasc Interv. vol. 3, ed United States, 2010, pp. 105-12. 